\title{
Mouvement brownien, turbulence et chaîne de Markov
}

\section{Brownian movement, turbulence and Markov chains}

\author{
C. Thirriot
}

Institut national polytechnique de Toulouse

Après avoir présenté une revue des approches de simulation stochastique de la turbulence depuis Brown, l'auteur montre les possibilités d'application des chaînes de Markov pour décrire le phénomène de dispersion turbulente et prendre en compte l'autocorrélation des fluctuations des vitesses.

After having presented a review concerning random turbulence simulation approaches since Brown, the author demonstrates the application possibilities of Markov chains so as to describe the phenomenon of turbulent dispersion and to take into account the autocorrelation of velocity fluctuations.

\section{Introduction et historique}

Il paraît que c'est justement en regardant le déplacement saccadé de particules dans un écoulement turbulent que le botaniste anglais Georges Brown eut l'idée en 1927 de ce qui allait être appelé le mouvement brownien.

II fallut cependant attendre 1905 pour qu'A. Einstein donne une présentation assez satisfaisante de la théorie de la dispersion liée à l'aspect statistique du mouvement brownien particulaire. Et l'exposé vraiment rigoureux ne viendra qu'en 1923 avec Wiener. Mais il s'agit là d'une approche lagrangienne: on suit le déplacement d'une particule qui a des aléas de vitesse en direction et en intensité.

A côté de l'approche statistique, il y a eu aussi le point de vue déterministe du mécanicien qui applique la loi fondamentale de la quantité de mouvement au choc ou à l'interaction de particules. Mais pour que les calculs soient de complexité supportable on introduit la discrétisation : la discrétisation dans le temps inhérente au mouvement brownien, la discrétisation des vitesses en intensité et direction et aussi la discrétisation des positions des particules qui ne pourront se trouver qu'aux nœuds d'un réseau. Ces hypothèses ont conduit au développement de la dynamique moléculaire.

Le Professeur Henri Cabannes (1980) donne un rapide historique de l'utilisation de distribution discrète de vitesse introduite dès 1890 par Maxwell pour un modèle de gaz à particules identiques et à six vitesses de même module suivant un repère cartésien tridimensionnel (Maxwell réussit ainsi à prévoir les propriétés et l'équation d'état d'un tel gaz). En 1901 sur la demande de Lord Kelvin, son assistant William Anderson utilise des nombres au hasard pour calculer 5000 collisions de particules avec des surfaces et 300 collésions intermoléculaires.

Dans la deuxième moitié $\mathrm{du} \mathrm{xx}^{*}$. siècle, les recherches et les résultats se multiplient surtout sur les gaz raréfiés, sur l'équation discrète de Boltzmann [Carleman (1957), Gross (1960)] mais aussi sur la propagation des ondes [Broadwell (1964), Gatignol (1965-1975) et Cabannes (1975)].

En 1972, Hardy et Pomeau retrouvent les équations d'Euler et de Navier Stokes en utilisant un modele plan à quatre vitesses.

Avec l'extension époustouflante des possibilités des ordinateurs, les recherches glissent durant les dernières années des études analytiques vers les simulations numéri- 
ques qui profitent d'abord à la dynamique moléculaire comme l'indique le courrier du CNRS 1985 "Image de la Physique " mais sont appliquées aussi de manière prometteuse à la mécanique des fluides (d'Humières, Pomeau et Lallemand 1985, Karweit 1985). Et récemment, un effort a été fait pour passer des plasmas ou des gaz moyennant denses aux liquides incompressibles en relayant le point de vue lagrangien du suivi des particules par le point de vue eulérien de distribution de champ aléatoire de vitesse en des points d'un réseau à deux et même trois dimensions.

Dans ce qui suit, nous reviendrons d'abord sur l'optique lagrangienne en examinant ce que peuvent apporter les chaînes de Markov discrètes puis nous donnerons un rapide aperçu sur les recherches en cours sur les simulations numériques à base de champs eulériens de vitesse.

\section{Approche particulaire stochastique Marko- vienne}

\subsection{Les prémisses}

Pour mettre en évidence ce qui revient spécifiquement à la turbulence nous considèrerons les déplacements désordonnés par rapport à l'écoulement uniforme : ces déplacements relatifs erratiques correspondent aux fluctuations turbulentes de vitesse.

La particule suit ce que André Craya appelait la démarche de l'homme ivre. Elle a des déplacements successifs, apparemment incohérents dans toutes les directions.

Comme pour les processus browniens, nous considèrerons les déplacements pendant des intervalles de temps successifs égaux $\Delta t$ petits.

Pendant l'intervalle $\Delta t$, le déplacement relatif $\Delta x$ permet de définir une vitesse moyenne fluctuante $v=$ $\Delta x / \Delta t$.

Pour simplifier l'exposé, nous allons d'abord considérer des fluctuations de vitesse "que suivent une seule direction qui peut être longitudinale ou transversale.

Pour avoir une image plus concrète nous prendrons des variations de vitesse orthogonale à la vitesse porteuse uniforme dans un écoulement plan. Le résultat de l'agitation turbulente sera alors la dispersion transversale.

\subsection{La discrétisation du champ de vitesse turbulente}

Suivant l'exemple de la dynamique moléculaire, nous considérerons deux cas très simples.

- La distribution binaire : la composante transversale de vitesse peut prendre deux valeurs opposées $v_{0}$ et $-v_{i 1}$.

- La distribution ternaire : la composante peut prendre successivement l'une des valeurs $v_{i}, 0$ et $-v_{0}$.

\subsection{L'introduction des chaînes de Markov binaires}

Bien que désordonnées, les fluctuations de vitesse ne sont pas forcément indépendantes et ce d'autant moins que l'intervalle de temps $\Delta t$ est petit compte tenu de l'inertie. Nous supposerons donc qu'à l'instant $t$ la vitesse turbu- lente dépend de sa valeur à l'instant $t-\Delta t$ en probabilité, par le jeu d'une chaîne de Markov discrète à deux états. Les probabilités conditionnelles constituent la matrice de Markov.
Vitesse à

l'instant $t-\Delta t$

$$
\begin{array}{r}
v_{0} \\
-v_{i}
\end{array}
$$

Vitesse à l'instant $t$

$\begin{array}{cc}v_{01} & -v_{11} \\ \alpha_{011} & \alpha_{01} \\ \alpha_{11} & \alpha_{11}\end{array} \mid$

On distingue les deux états binaires, 0 si la vitesse est $v_{i}, 1$ si la vitesse est $-v_{0}$.

Les probabilités conditionnelles $\alpha_{1 j}$ sont telle que:

$$
\alpha_{n j}+\alpha_{n 1}=1
$$

Si la dispersion transversale est symétrique, on peut admettre que $\alpha_{0 \mid 1}=\alpha_{11}$, la probabilité de persistance dans un état est supposée la même pour les deux états.

S'il y a une contrainte (paroi, fort gradient de vitesse de translation longitudinale) on pourrait faire intervenir une dissymétrie sur ces coefficients mais ceci correspondrait à une déflexion de la trajectoire moyenne.

En effet, les probabilités marginales ou inconditionnelles sont :

et

$$
\begin{gathered}
F_{0}=\alpha_{10} /\left(\alpha_{01}+\alpha_{10}\right) \\
F_{1}=\alpha_{011} /\left(\alpha_{01}+\alpha_{10}\right)=1-F_{0}
\end{gathered}
$$

Donc, si on admettait une différence dans les probabilités conditionnelles $\alpha_{0 i t}$ et $\alpha_{11}$, il s'en suivrait une déflexion de la trajectoire. Autant changer alors la vitesse porteuse moyenne pour se retrouver dans la condition de moyenne nulle des vitesses turbulentes.

$$
\text { Ainsi } \quad F_{0}=F_{1}=1 / 2
$$

Qu'y a t'il donc de changé par rapport à l'hypothèse d'indépendance? C'est l'introduction d'une corrélation entre les vitesses successives.

\subsection{L'expression du coefficient d'autocorrélation dans les chaînes de Markov binaires}

Avec l'espérance nulle de la composante turbulente la matrice binaire se présente sous la forme

$$
\mid \begin{aligned}
& \alpha \\
& 1-\alpha
\end{aligned}
$$

$$
\begin{aligned}
& 1-\alpha \\
& \alpha
\end{aligned} \mid
$$

Soit $t=k \Delta t$ la date d'évaluation de la vitesse $v_{k}=v(t)$. $\mathrm{Si} N$ est le nombre d'observation, par définition le coefficient de corrélation est :

$$
\rho=\left(\sum_{k=1}^{N-1} v_{k} v_{k+1}\right) /\left[(N-1) \sigma_{i}^{2}\right]
$$

$\sigma_{i}$ étant l'écart type de la vitesse fluctuante soit $\sigma_{i}=v_{i t}$. De manière générale :

$\sum_{k=1}^{i-1} v_{k} v_{k+1}=(N-1)\left\{F_{0}\left(\alpha_{403}-\alpha_{01}\right)+F_{1}\left(\alpha_{11}-\alpha_{10}\right)\right\}$

Ce qui devient ici : 


$$
\sum_{k=1}^{N-1} v_{k} v_{k+1}=(N-1)(2 \alpha-1)
$$

D'où

$$
p=2 \alpha-1
$$

Au passage signalons que $\rho$ est aussi la deuxième valeur propre de la matrice de Markov, l'autre étant évidemment 1 .

\subsection{L'estimation de la dispersion turbulente}

Considérons une particule à l'instant 0 . A l'instant $t=N \Delta t$, elle marquera un écart par rapport à la trajectoire idéale rectiligne uniforme égal à :

$$
Y_{s}=\sum_{k=1}^{x} v_{k} \Delta t
$$

Cet écart est bien sûr de moyenne nulle mais ce qui va le caractériser c'est la valeur moyenne quadratique $\sigma_{r}$.

Le calcul de la variance de la somme de valeurs booléennes liées par une chaîne de Markov est peut être quelque chose de classique dont nous n'avons pas su trouver la référence. Peut être est-ce aussi un exercice ludique que de l'établir. C'est ce que nous avons fait il y a quelques années (Thirriot 1983). Nous avons obtenu :

$\operatorname{Var}\left(Y_{N}\right)=[N+(2 N \rho /(1-\rho))$

$$
\left.\left.-\left(2 \rho\left(1-\rho^{v}\right)\right) /\left(N(1-\rho)^{2}\right)\right)\right] v_{i i}^{2} \Delta t^{2}
$$

Si $N$ est très grand:

$$
\sigma_{Y N} \rightarrow v_{0} \sqrt{(N(1+\rho) /(1-\rho))} \Delta t
$$

Si les fluctuations successives de vitesse sont indépendantes

$$
\sigma_{r N}=v_{0} \sqrt{N} \Delta t
$$

On retrouve là, un résultat classique de la théorie de la dispersion

$$
\sigma_{Y}=v_{0} \cdot \sqrt{\Delta t \cdot t}
$$

S'il y a une corrélation, la dispersion turbulente est plus importante.

\subsection{L'exploitation du modèle markovien binaire}

Quand $N$ devient très grand l'expression de l'écart type a même forme que les vitesses turbulentes successives soient indépendantes ou non: il n'est pas possible de distinguer l'influence de $\rho$ de celle de l'intensité turbulente. Cette distinction est en principe possible si $N$ est petit.

L'écart relatif sur la variance est :

$$
\left.\Delta \operatorname{Var}\left(Y_{N}\right) / \operatorname{Var}\left(Y_{N}\right)\right)=\left(2 \rho\left(1-\rho^{*}\right)\right) /\left(1-\rho^{2}\right) N^{2}
$$

L'écart relatif sur l'écart type est donc approximativement

$$
\left(\Delta \sigma_{Y} / \sigma_{Y N}\right) \simeq \rho /\left(1-\rho^{2}\right) N^{2}
$$

Il faut donc vraiment une forte corrélation pour que l'influence de $\rho$ soit décelable sur le graphe d'évolution du coefficient de dispersion turbulente.

Par contre si on a pu mesurer à part l'intensité turbulente, l'observation de la dispersion permet alors d'obtenir le coefficient de corrélation.

\subsection{Le retour au point de vue eulérien}

On peut aussi utiliser l'image de la matrice de Markov binaire pour décrire la liaison entre vitesses turbulentes successives en un point fixe de l'espace et construire l'autocorrélogramme.

Reprenons la matrice de transition en l'écrivant sous la forme de somme de matrices propres aux deux valeurs propres 1 et $\rho$

$T=\left|\begin{array}{cc}\alpha & 1-\alpha \\ 1-\alpha & \alpha\end{array}\right|=\left|\begin{array}{cc}1 / 2 & 1 / 2 \\ 1 / 2 & 1 / 2\end{array}\right|+\rho\left|\begin{array}{rr}1 / 2 & -1 / 2 \\ -1 / 2 & 1 / 2\end{array}\right|$

Les deux matrices propres sont orthogonales ce qui permet d'écrire :

$$
T^{N}=\left|\begin{array}{ll}
1 / 2 & 1 / 2 \\
1 / 2 & 1 / 2
\end{array}\right|+\rho^{\prime}\left|\begin{array}{rr}
1 & -1 \\
-1 & 1
\end{array}\right|
$$

Le coefficient d'autocorrélation d'ordre $N$ est :

$$
\left.\rho_{N}=\left[\left(\sum_{k=1}^{1 / x-1} v_{k} v_{k+1}\right) /\left((M-N) \sigma v^{2}\right)\right)\right]=\rho^{v}
$$

L'autocorrélogramme est donc exponentiel.

\subsection{De la persistance d'une vitesse à l'appréciation de la taille d'un tourbillon}

Inutile de revenir sur l'entomologie des tourbillons: les gros tourbillons pleins d'énergie cinétique vont nourrir les petits tourbillons voraces qui dissipent l'énergie.

Nous allons essayer de nous servir du modèle markovien pour avoir une idée sur la fonction de répartition en taille des tourbillons. Bien entendu, comme dans tout modèle, il y aura dans ce qui suit une part de convention qui peut être considérée comme arbitraire.

Notre hypothèse de modélisation est la suivante: comme les éléments de vitesse turbulente sont opposés, nous dirons que la taille d'un tourbillon est grosso modo de l'ordre de grandeur de la distance afférente à une séquence de vitesse de même signe, ce qui revient à admettre que deux tourbillons adjacents ou successifs tournent en sens contraire. Bien sûr, la critique est aisée de cette schématisation à la hussarde. Mais acceptant temporairement cette hypothèse, voyons les conséquences.

L'étude des séquences de même état est classique dans les processus markoviens. Nous-mêmes, nous nous en sommes beaucoup servi dans l'examen des séries pluviométriques (Thirriot 1983a, Arnaud 1985). Nous ne reprendrons pas ici en détail les calculs.

La probabilité conditionnelle d'avoir une vitesse positive sur un intervalle de temps $\Delta t$ est $\alpha_{10}=1-\alpha$.

La probabilité de vitesse positive sur deux intervalles en suivant est $\alpha_{10} \alpha_{0 \mid \zeta}=\alpha(1-\alpha)$.

La probabilité de vitesse positive sur $N$ intervalles successifs est $\alpha_{10} \alpha_{6 i 1}^{v-1}=\alpha^{v-1}(1-\alpha)$.

La fonction de répartition des séquences de vitesse positive est donc :

$$
F(N)=1-\alpha^{*}=1-((1+\rho) / 2)^{N}
$$


L'espérance mathématique de la longueur de séquence de vitesse positive est :

$$
\begin{aligned}
L=\sum_{i=1}^{\infty} N \alpha_{10} \alpha_{i n i}^{i-1} / \sum_{i=1}^{\infty} \alpha_{i t} \alpha_{i n i}^{i-1} & \\
& =1 /\left(1-\alpha_{i n i}\right)=1 /(1-\alpha)=2 /(1-\rho)
\end{aligned}
$$

On pourrait de même obtenir l'écart type de la taille des tourbillons en calculant la variance des longueurs de séquences

$$
\sigma_{l}=\sqrt{\alpha_{10}} /\left(1-\alpha_{111}\right)
$$

D'où le coefficient de variation

$$
C_{:}=\sigma_{l} / L=\sqrt{(1+\rho) / 2}
$$

\subsection{L'approximation du spectre de l'énergie turbulente}

Voyons à quelles conclusions énergétiques aboutit notre hypothèse de répartition de population de tourbillons.

Admettons que le rayon du tourbillon soit proportionnel à la longueur de séquence de vitesse de même sens, $r \sim N$.

Soit $L$ la longueur du tourbillon. Si on le suppose cylindrique, sa masse est $m=\rho \pi r^{2} L$ son moment cinétique $C=m r^{2} w / 2$ où $w$ est la vitesse de rotation et son énergie cinétique $E=m r^{2} w^{2} / 4$.

Il est maintenant classique de parler de la déformation des tourbillons par étirement (Haminh et Chassaing 1980). Et l'étirement d'un tourbillon dans un sens va provoquer l'étirement de deux autres tourbillons dans les directions orthogonales et il y a ainsi prolifération des étirements suivant l'arbre généalogique de Bradshaw. Et ceci à partir d'un premier tourbillon qui, à peu près aligné avec l'axe de la déformation moyenne, va pomper de l'énergie au mouvement moyen, énergie qui sera transmise de proche en proche aux tourbillons orthogonaux étirés. Car dans l'élongation, un tourbillon accumule plus d'énergie cinétique. En effet si l'on admet que dans l'étirement masse et moment cinétique sont conservés, $w$ varie comme $L$ et $E$ varie comme $w$ (ou $L$ ). Et le supplément d'énergie cinétique doit être fourni par les tourbillons plus gros : c'est la cascade de Kolmogorov alimentée en début de chaîne par le mouvement moyen.

Dans le cas d'une turbulence homogène stationnaire, la situation énergétique va être représentée par le spectre de l'énergie. Pour la vitesse de rotation $w$ (liée à la fréquence du tourbillon), la proportion en nombre est déterminée par la fonction de répartition $F(N)$ puisque $N$ est proportionnel à $r$ et que $w$ évolue comme $1 / r$.

Avec l'empilement des hypothèses ci-dessus (d'une part sur la distribution des tailles de tourbillons, d'autre part sur l'étirement) on arriverait à une fonction de densité d'énergie en fonction de la fréquence (ou de $w$ ).

Il nous semble raisonnable de penser que les tourbillons étirés deviennent fragiles, cassent et sont fractionnés. Admettons qu'après brisure la longueur des tourbillons soient de l'ordre de grandeur du rayon. Le volume du tourbillon élémentaire après cassure est proportionnel à $r^{3}$ ou $L^{3}$. La proportion en nombre d'après le schéma markovien en prenant l'extension de $r$ réel au lieu de $N$ entier est telle que :

$$
d F / d r=|\log \alpha| \exp (-r|\log \alpha|)
$$

L'énergie du tourbillon élémentaire est proportionnelle à $\rho r^{5} w^{2}$, ou encore proportionnelle à $r$ compte tenu des hypothèses de l'étirement (à $m$ et $C$ constants).

Dans la distribution de l'énergie sera donnée par la densité de la fonction de répartition :

$$
d G / d r=A r|\log \alpha| \exp (-r|\log \alpha|)
$$

ou $G=1-(1+r|\log \alpha|) \exp (-r|\log \alpha|)$

Soit encore si on considère le spectre de l'énergie en fonction de la fréquence puisque $w^{2} r$ est considéré comme constant dans l'étirement.

$$
\begin{aligned}
G(w)=1-\left(1+\left(r_{0} w_{0}^{2} / w^{2} \mid \log \alpha i\right)\right. \\
\cdot \exp \left(\left(-r_{01} w_{0}^{2} / w^{2}\right)|\log \alpha|\right)
\end{aligned}
$$

D'où la densité d'énergie :

$$
\begin{aligned}
& \varphi(w)=-d G / d w=\left(2 r_{0}^{2} w_{0}^{4} / w^{5}\right)|\log \alpha|^{2} \\
& \text {. } \exp \left(\left(-r_{0}\left(w_{i}^{2} / w^{2}\right)|\log \alpha|\right)\right. \\
& \text { ou } \\
& \varphi(w)=2 r_{0}^{2}\left(w_{0}^{4} / w^{5}\right)|\log \alpha|^{2} \alpha^{-r_{10}}\left(w_{0 i}^{2} / w^{2}\right)
\end{aligned}
$$

Posons

$$
w / w_{0}\left(r_{0}|\log \alpha|\right)^{1 / 2}=\Omega
$$

Il vient que $f=\varphi / \varphi_{0}=1 / \Omega^{5} \exp \left(-1 / \Omega^{2}\right)$.

Le graphe $f(\Omega)$ fait apparaître un maximum pour $\Omega=2 / 5$ avec des variations de part et d'autre plus rapides qu'il n'apparaît dans les résultats expérimentaux ou dans la théorie de Kolmogorov.

\begin{tabular}{|c|c|c|c|c|c|c|}
\hline$\omega$ & 0,2 & 0,3 & 0,4 & 0,5 & $2 / 5$ & 0,8 \\
\hline$Q$ & $4,410^{-8}$ & 0,006 & 0,19 & 0,59 & 0,82 & 0,64 \\
\hline
\end{tabular}

\begin{tabular}{|c|c|c|c|c|c|}
\hline$\omega$ & 1 & 1,5 & 2 & 5 & 10 \\
\hline$Q$ & 0,32 & 0,084 & 0,024 & $310^{-4}$ & $10^{-5}$ \\
\hline
\end{tabular}

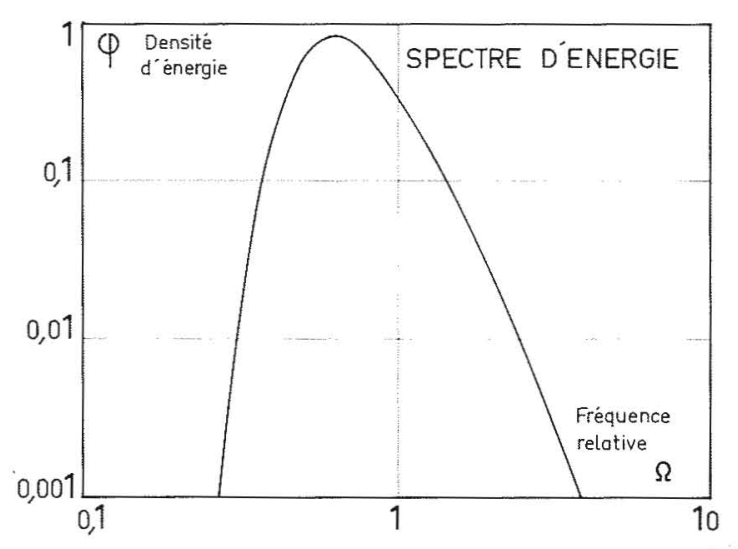

Le modèle examiné conduirait du point de vue énergétique à un modèle passe bande, les fréquences de coupure (approximative) dépendant de la probabilité conditionnelle $\alpha$. Mais évidemment bien des hypothèses admises dans le cheminement ci-dessus sont fort contestables 
(relation entre longueur et rayon des tourbillons, passage d'une fonction de répartition pour longueur de séquence discrète à un graphe à variable continue, le rayon, et bien sûr convention d'évaluation de la grosseur des tourbillons sur le changement de sens de la vitesse).

Si l'on tenait à tout prix à donner une interprétation physique du graphe obtenu on pourrait dire que les gros tourbillons après formation s'engraissent énergétiquement aux frais du mouvement moyen en s'étirant tout en créant par cisaillement, d'autres structures. Pour l'ensemble, l'énergie cinétique croit tant que les créations et l'effet d'accélération l'emportent sur la dissipation pour les fréquences modérés. Puis au-delà d'une fréquence, les petits tourbillons véloces dissipent plus qu'ils ne transmettent d'énergie.

\subsection{De l'effet de l'agrégation}

Compte tenu de la définition des composantes turbulentes comme moyenne de la vitesse fluctuante dans l'intervalle de temps $\Delta t$, l'agrégation sur des intervalles plus grands $p \Delta t$ est aisée. On revient à la considération des sommes des déplacements $\Delta Y_{\text {r. }}$.

Sur l'intervalle $p \Delta t$, la moyenne de la vitesse turbulente est :

$$
W_{k}=\sum_{1=11}^{1-1} v_{k+1} / p
$$

Bien entendu pour $p$ fini, la distribution des nouvelles vitesses $W$ est encore discrète mais plus diversifiée. Par exemple pour $p=2$ les vitesses sont 1,0 et -1 avec les probabilités marginales respectives $1 / 4,1 / 2$ et $1 / 4$. On a affaire à la distribution binomiale qui pour $n$ grand va tendre vers la distribution normale ce qu'on aurait pu induire de suite du théorème «limite centrale" (qui affirme que sous des conditions somme toute peu contraignantes toute somme de variables aléatoires même issues de populations différentes admet la loi normale comme fonction de répartition).

Evidemment la matrice carrée de Markov va avoir la taille du vecteur des vitesses possibles. On va ainsi perdre la simplicité de la matrice binaire pour la richesse des processus multiclasses, richesse assortie d'une complication dans le calcul.

A titre d'exemple pour $p=2$, la matrice de transition construite à partir du processus binaire sera la suivante :

$$
\begin{array}{l|lll|}
\multicolumn{1}{c}{1} & 0 & -1 \\
1 & \alpha^{2} & 1-\alpha & \alpha(1-\alpha) \\
0 & \alpha / 2 & 1-\alpha & \alpha / 2 \\
-1 & \alpha(1-\alpha) & 1-\alpha & \alpha^{2}
\end{array}
$$

Nous examinerons un peu plus loin les propriétés de cette corrélation dans le cadre plus général d'une matrice de transition ternaire.

\subsection{L'utilisation d'une distribution discrète à trois vitesses}

Nous admettrons que les fluctuations lagrangiennes trans- versales puissent être représentées par trois valeurs de vitesses qui par raison de symétrie évidente seront $v_{\omega}, 0$ et $-v_{i j}$

\subsubsection{Expression de la matrice de transition:}

Pour une particule, les probabilités de passage d'une valeur de vitesse à une autre à l'instant suivant sont données par la matrice de transition suivante:
Etat de vitesse

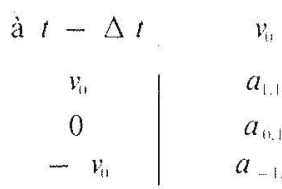

Etat de vitesse à $t=k \Delta t$

$\begin{array}{cc}0 & -v_{n 1} \\ a_{1, n} & a_{1, \ldots, 1} \\ a_{0, n} & a_{n, \ldots 1} \\ a_{-1, n} & a_{-, \ldots 1}\end{array} \mid$

Par raison de symétrie, à partir de l'état médian il y a même probabilité d'atteindre un état extrême.

$$
a_{10,1}=a_{1, \ldots 1}=\left(1-a_{1+1}\right) / 2
$$

Toujours par raison de symétrie on peut permuter les rôles des états 1 et -1 , donc:

$$
\begin{aligned}
& a_{-1,-1}=a_{1 !} \\
& a_{1,1}=a_{-1.11} \\
& a_{1,-1}=a_{1,1}
\end{aligned}
$$

En définitive, il ne reste donc que trois paramètres:

$$
a_{i n, 1}, a_{1 ;} \text { et } a_{!, 1}
$$

La matrice de transition est :

$$
T=\left|\begin{array}{lll}
a_{11} & a_{111} & 1-a_{11}-a_{1+1} \\
\left(1-a_{111}\right) / 2 & a_{01 !} & 1-a_{011} / 2 \\
1-a_{11}-a_{11} & a_{1+1} & a_{11}
\end{array}\right|
$$

\subsubsection{Recherche des valeurs propres}

Une valeur propre $\lambda_{i}$ est évidemment égale à l'unité. Les autres sont :

$$
\begin{aligned}
& \lambda_{2}=a_{11}-a_{1,-1} \\
& \lambda_{3}=a_{51}-a_{1,0}
\end{aligned}
$$

Un calcul scolaire permet d'obtenir alors les systèmes de vecteurs propres associés aux valeurs propres.

Pour $\lambda_{1}=1, \quad \vec{u}_{1}=(\beta, \gamma, \beta)$

$$
\begin{array}{ll}
\text { avec } & \gamma=\sqrt{1-2 \beta^{2}} \text { et } \beta / \gamma=1-a_{00} / 2 a_{16} \\
\text { Pour } \lambda_{2} & \vec{u}_{2}=(1 / \sqrt{2}, 0,-1 / \sqrt{2}) \\
\text { Pour } \lambda_{3} & \vec{u}_{3}=(1 / \sqrt{6},-2 / \sqrt{6}, 1 / \sqrt{6})
\end{array}
$$

Soit la matrice $T$ formée des 3 vecteurs propres, nous pouvons montrer que :

$$
\begin{gathered}
T^{*}=\sigma^{-1} T^{* x} \sigma \\
\text { avec } T^{*}=\left|\begin{array}{ccc}
\lambda_{1} & 0 & 0 \\
0 & \lambda_{2} & 0 \\
0 & 0 & \lambda_{3}
\end{array}\right| \\
\text { D'où : } T^{N}=T_{1}+\lambda_{2}^{y} T_{2}+\lambda_{3}^{y} T_{3}
\end{gathered}
$$


avec

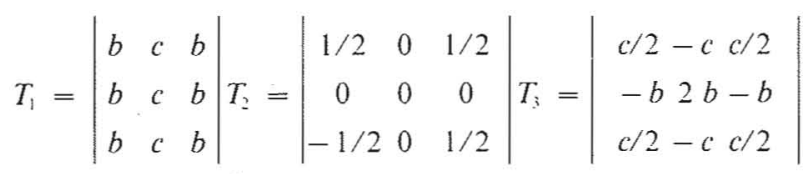

où

$$
b=\beta /(\gamma+2 \beta), c=\gamma /(\gamma+2 \beta)=1-2 b
$$

$b$ et $c$ sont les probabilités marginales ou inconditionnelles

\subsubsection{Signification physique des valeurs propres $\lambda_{2}$ et $\lambda_{3}$}

Puisque l'intérêt des chaines de Markov discrètes est de prendre en compte les corrélations entre états successifs, nous avons tenté le calcul de coefficients de corrélation.

a) Calcul du coefficient d'autocorrélation entre les fluctuations de vitesse

$$
\begin{gathered}
\rho=E\left(v_{k}, v_{k+1}\right) / E\left(v_{k}^{2}\right) \\
\text { Evidemment } E\left(v_{k}\right)=0 \\
E\left(v_{k}, v_{k+1}\right)=2 b\left(a_{11}-a_{1-1}\right)
\end{gathered}
$$

et

$$
E\left(v_{k}^{2}\right)=2 b
$$

Donc $\rho=a_{11}-a_{1,-1}=\lambda_{2}$

Pour le processus binaire, nous avons aussi obtenu le coefficient de corrélation égal à la deuxième valeur propre. Est-il possible d'envisager la généralisation de ce résultat pour une matrice de transition d'ordre plus élevé ?

b) Calcul du coefficient d'autocorrélation de l'énergie cinétique fluctuante

Comme la fusion des états $v_{0}$ et $-v_{0}$ ramène à une matrice binaire, nous avons pensé à considérer l'énergie cinétique $E$ propre à la composante fluctuante de la vitesse. $E$ prend les valeurs 0 et $v_{0}^{2} / 2$ corrélés par la matrice binaire suivante.

$$
\begin{aligned}
& \text { Etat à } t-\Delta t=(k-1) \Delta t \quad \text { Etat à } t=k \Delta t
\end{aligned}
$$

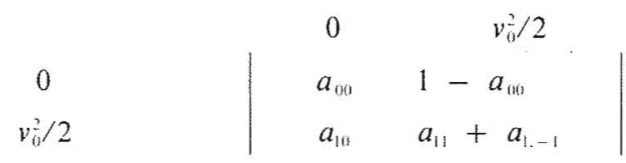

Le coefficient de corrélation de cette matrice binaire est :

$$
\rho^{\prime}=a_{00}-a_{110}=\lambda_{3}
$$

\subsubsection{Identification du modèle markovien ternaire comme image de la turbulence}

Il est simple d'identifier les paramètres de la matrice de transition ternaire à partir des observations des fluctuations de la vitesse turbulente après discrétisation en 3 classes dont l'étude statistique fournit :

b : la probabilité marginale de la classe de vitesse $v_{0}$

$\rho$ : le coefficient d'autocorrélation d'ordre 1 de la composante fluctuante. $\rho^{\prime}:$ le coefficient d'autocorrélation de l'énergie cinétique fluctuante.

Bien sûr avec trois classes, il ne faut pas prétendre représenter toute la richesse de la réalité d'une turbulence même homogène et isotrope. Mais on peut tout de même obtenir une information sur le coefficient de dispersion turbulente par le biais du calcul de l'écart type du déplacement cumulé transversal.

Reste la question délicate du découpage en classes qui va jouer à la fois sur $v_{10}$ et sur la probabilité marginale b (ou c).

Coupons à $-v_{s}$ et $+v_{s}$. En admettant la symétrie de la fonction de répartition $F(v)$ par rapport à 0 , la moyenne entre $-v_{s}$ et $+v_{s}$ est nulle. La probabilité marginale est :

$$
\int_{-4}^{n} f(v) d v \quad \text { avec } f(v)=d F(v) / d v
$$

ou $\quad c=2(F(v)-F(0))$

Ensuite $v_{0}$ est l'espérance mathématique pour $v>v_{s}$. Mais on pourrait tout aussi bien prendre un critère de conservation de l'intensité turbulente.

\subsection{L'utilisation de processus de Markov discrets d'ordre supérieur}

L'observation des fonctions d'autocorrélation eulérienne de la turbulence montre que très souvent le coefficient d'autocorrélation qui semble marquer d'abord une décroissance exponentielle lorsque l'intervalle s'accroît, peut présenter ensuite une plage de légères valeurs négatives avant de s'éteindre.

Nous avons montré (au moins de manière approchée) qu'un tel autocorrélogramme pouvait être obtenu à partir d'un processus discret binaire de Markov d'ordre supérieur. Par exemple pour un processus d'ordre 2, le corrélogramme est la somme de deux exponentielles qui peut très bien s'ajuster à la présence d'une partie négative.

Avec des processus d'ordre 2 ou même 3 , on tient ainsi mieux compte de la mémoire du phénomène c'est-à-dire de l'inertie des particules fluides.

\subsection{La considération de fluctuations tridimensionnel- les}

Une première généralisation simple mais pas complètement rationnelle consiste à dire que l'on peut considérer des fluctuations discrètes suivant trois directions orthogonales sans faire intervenir de corrélation entre les fluctuations de vitesse ayant lieu dans deux directions différentes. Les résultats obtenus précédemment sont alors directement applicables en particulier pour juger de la dispersion tridimensionnelle.

Une extension plus satisfaisante consiste à faire intervenir une corrélation entre les composantes fluctuantes suivant les trois directions mais alors même dans les cas binaires la matrice sera de dimension six. Et bien que la turbulence soit essentiellement un phénomène tridimensionnel, on peut à titre d'exercice, examiner le cas d'un écoulement plan pour lequel la matrice sera de taille quatre. 


\subsection{Les perspectives offertes par d'autres approches stochastiques}

On peut ainsi revenir à des distributions continues de vitesse soit en utilisant les méthodes de régression linéaire, soit en exploitant des procédés hybrides tels ceux que je fis utiliser à Michel Arnaud pour la modélisation de la pluviométrie quotidienne.

Les pistes de la modélisation stochastique sont nombreuses mais les images obtenues ou recherchées ne seront jamais que des êtres analogiques qui présenteront quelques uns des traits caractéristiques de la turbulence mais ne seront en définitive qu'une illusion sans fondement physique.

Ce fondement physique est recherché dans les modèles issus de la dynamique moléculaire et les simulations sur réseaux mais est-il plus tangible?

\section{Les simulations de la turbulence sur réseaux}

Au cours des dernières décennies, les tentatives furent multiples. Parmi les plus récentes nous allons examiner brièvement celle de Dominique d'Humières, Yves Pomeau et Pierre Lallemand de l'Ecole Normale Supérieure de Paris et celle de Mickaël Karweit de l'Université Johns Hopkins de Baltimore. Les premiers se sont attachés à reproduire les allées de tourbillons de Von Karman derrière différents obstacles (plaque, disque) placés dans un gaz compressible. Il ne s'agit donc pas à proprement parler de turbulence mais de mouvement de grosses structures tourbillonnaires encore bien organisées. Le chercheur américain lui s'est intéressé à l'étude de la dispersion dans des écoulements incompressibles dont la simulation constitue une difficulté importante par rapport à la modélisation de gaz compressibles.

Le point commun entre les auteurs français et américain est l'utilisation de vitesse discrète de même module et de déplacement synchronisé de particules.

En ce qui concerne le dessin du réseau, les chercheurs français après avoir essayé des réseaux plans orthogonaux comme Karweit, se sont tournés vers les réseaux hexagonaux à base de triangles équilatéraux. Sur de tels réseaux, six particules peuvent converger vers un nœud où se trouvent éventuellement des particules immobiles. Le choix des lois de collision entre particules va déterminer les propriétés du fluide. D'après les auteurs, une analyse théorique permet de montrer que les valeurs moyennes, masse volumique et quantité de mouvement, vérifient les équations de continuité et de Navier-Stokes ce qui n'étaient pas possible avec le réseau à mailles carrées encore utilisées par Karweit.

Ces lois de collisions font intervenir des changements de directions qui surprennent d'abord mais pourraient s'expliquer dans certains cas du point de vue mécanique par le choc de boules de billard ou de pétanque aux centres de gravité déportés au moment de la rencontre mais il ne faut peut être pas vouloir pousser l'analogie à fond.

Pour Karweit, les lois de collisions deviennent des lois de remplacement particule pour particule puisqu'il faut assurer l'incompressibilité du fluide et pour ce faire, le chercheur américain construit des tables de correspondance entre les vecteurs vitesses " entrée " possibles et les vecteurs « sortie » puisqu'il ne peut y avoir accumulation. Cette contrainte d'incompressibilité amène d'ailleurs Karweit à considérer des champs eulériens aléatoires de vitesse au lieu de répartitions lagrangiennes des vitesses attachées aux particules comme cela serait possible dans un fluide compressible. Il s'ensuit donc des précautions délicates pour la construction des champs eulériens assurant toutes les compatibilités aux nœuds adjacents.

Comme on peut le sentir, les choses ne sont pas si simples que pourrait le laisser croire la rusticité des méthodes de Monte Carlo. Ou bien, on travaille sur des gaz somme toute peu denses mais avec la satisfaction de la bonne vérification des équations de Navier Stockes en moyenne dans les nuages de points. Ou bien, on utilise des liquides incompressibles mais la démarche nous paraît alors purement stochastique. Dans les deux cas il nous semble qu'il s'agisse encore plus d'analogie ou d'image de la physique que d'une photographie de la réalité des écoulements turbulents. Ajoutons que la plupart des simulations ont été réalisées en réseau bidimensionnel alors que les auteurs reconnaissent volontiers que la turbulence est un phénomène tridimensionnel.

Bien sûr, il faudrait parler aussi du coût du calcul compte tenu de la nécessité du nombre énorme de répétitions d'essais en des points qui pullulent pour assurer des moyennes statistiques sûres. D'après J.C. Adam (1985) et G.A. Bird (1978) pour des calculs simulaires de dynamique moléculaire avec les machines vectorielles les plus rapides effectuant près d'un milliard d'opérations à la seconde, il faut environ une seconde par pas de déplacement pour suivre dix mille particules (ce qui est encore peu du point de vue statistique). Or, il faut suivre les déplacements pendant mille à dix mille pas.

Mais la solution est dans l'utilisation de calculateurs spécialisés bon marché. "Un million d’ordinateurs pour démontrer la mécanique des fluides " titre le journal Le Monde en 1986 dans un entrefilet «Sciences ». Il suffirait d'assembler des puces informatiques qui effectueraient les calculs simples afférents aux lois de collision ou de remplácement en chacun des sommets du réseau. Déjà en 1978, Bird proposait de faire fonctionner sans arrêt jour et nuit un tout petit ordinateur disposant d'un processeur très rapide et il estimait à cinquante francs le coût du calcul d'un million de collisions, coût qui s'est bien sûr effondré depuis. 


\section{Conclusion}

La simulation stochastique, qu'elle soit analytique ou numérique, donne très vite des résultats qui ont agréablement un comportement analogue à la réalité. Phénomène de dispersion bien approché depuis Einstein, tourbillons alternés très évocateurs produits par les nuages de particu- les lancées dans l'ordinateur. De nouveaux progrès sont à l'horizon avec la coordination fructueuse de démarches différentes des champs eulériens aléatoires correlées et de la dynamique des particules vérifiant en moyenne les équations de Navier-Stokes. Mais il nous semble qu'actuellement ces approches stochastiques sont encore plus une aide à la réflexion ou à la digestion des résultats d'observation qu'un outil de prévision dans les projets industriels.

\section{Bibliographie}

ADAM J.C., (1985). - Simulations particulaires en physique des plasmas. Le courrier du CNRS. Image de la Physique, supplément au $\mathrm{n}^{\circ} 59$, pp. 22-27.

Arnaud M. (1985). - Contribution à l'étude stochastique markovienne des précipitations dans le bassin Adour-Garonne. Thèse présentée à l'Institut national polytechnique de Toulouse.

BIRD G.A., (1978). - Monte Carlo Simulation of gas flows. Ann. Rev. Fluid Mech., n"10, pp. 11-31.

CABANNES H., (1980). - The discrete Boltzmann equation (theory and application). Lectures notes given at the University of California Berkeley. College of Engineering University of California Berkeley.

Chassaing D., Haminh H. (1980). - Turbulence. Introduction à l'analyse physique et modélisation mathématique. Cours polycopié ENSEEIHT INP Toulouse.

Cox D.R., MiLler H.D., (1965). - The theory of stochastic processes. Methuen and CO, LONDON, p. 203.

EINSTEIN A., (1905). - Uber die von der molekularkinetishen Theorie der Warme geforderte Bewegung von in ruhenden Flussigkeiten suspendierten Teilchen. Ann. Jd. Phisik, 17, p. 549 .

Gatignol R. (1975). - Théorie cinétique des gaz à répartition discrète de vitesses. Lecture Notes in Phys., vol. 36, Springer Verlag, Heidelberg.

HaminH H. (1987). - Notes manuscrites sur la dynamique de la turbulence (communication personnelle).
Hansen J.P., LeVesque D. (1985). - La dynamique moléculaire. Le courrier du CNRS. Image de la Physique, supplément au n" 59, pp. 7-11.

Humieres D., Pomeau Y., Lallemand P. (1985). - Simulation d'allées de Von Karman bidimensionnelles à l'aide d’un gaz sur réseau. CR Acad. Sc. Paris, t. 301, Série II, n" 20, pp. 1391-1394.

KELVIN L., (1901). - Nineteenth century clouds over the dynamical theory of heat and light. Philos. Mag. 2, pp. 1-40.

Kemeny J.G., SNell J. (1965). - Finite Markor chains. D. Van Nostrand Company.

KurWaIT M., (1985). - Random incompressible motion on two and three dimensional lattices and its application to the walk on a random field. In Frontiers in fluid mechanics, ed. Davis S.H., Lumley J.L., Springer Verlag, pp. 22-39.

LUMley J.L., CORRSIN S. (1959). - A random walk with both Lagrangian and Eulerian Statistics. Advances in Geophysics, 6 , pp. $179-184$.

TARNOVSKI D., (1986). - Les supercalculateurs bientôt démodés ? La Recherche, février, Vol. 17, pp. 272-273.

THIRriot C. (1983a). - Etude de la variabilité des séries suivant une chaine de Markor binaire. Rapport interne IMFT. CT : 584 , juillet.

THIRRIOr C., (1983b). - Matrice de Markor temaire pour une schématisation naîve de la dispersion turbulente. Rapport interne IMFT CT : 589, octobre. 